This is an author version of the contribution published in Communications in Algebra

The definitive version is available at http://www.tandfonline.com/doi/abs/10.1080/00927872.2014.897565

DOI:10.1080/00927872.2014.897565 


\title{
A factorization theorem for topological abelian groups
}

\author{
Dikran Dikranjan \\ dikran.dikranjan@uniud.it \\ Dipartimento di Matematica e Informatica, \\ Università di Udine, \\ Via delle Scienze, 206 - 33100 Udine, Italy
}

\author{
Anna Giordano Bruno \\ anna.giordanobruno@uniud.it \\ Dipartimento di Matematica e Informatica, \\ Università di Udine, \\ Via delle Scienze, 206 - 33100 Udine, Italy
}

Dedicated to the 65th birthday of Marco Fontana

\begin{abstract}
Using the nice properties of the w-divisible weight and the $\mathrm{w}$-divisible groups, we prove a factorization theorem for compact abelian groups $K$; namely, $K=K_{\text {tor }} \times K_{d}$, where $K_{\text {tor }}$ is a bounded torsion compact abelian group and $K_{d}$ is a w-divisible compact abelian group. By Pontryagin duality this result is equivalent to the same factorization for discrete abelian groups proved in 9 .
\end{abstract}

\section{Introduction}

The main concept used in this paper is the following one, introduced and studied in [5]. We denote by $w(G)$ the weight of a topological group $G$, while $\mathbb{N}_{+}$indicates the set of positive integers.

Definition 1.1. Let $G$ be a topological abelian group. The divisible weight of $G$ is

$$
w_{d}(G)=\inf \left\{w(m G): m \in \mathbb{N}_{+}\right\} .
$$

A topological abelian group $G$ is $w$-divisible if $w(G)=w_{d}(G) \geq \omega$ (i.e., $w(m G)=w(G) \geq \omega$ for every $m \in \mathbb{N}_{+}$).

In particular, an infinite discrete abelian group $G$ is w-divisible if and only if $|m G|=|G|$ for every $m \in \mathbb{N}_{+}$.

This definition is different from the original definition from [5], where instead of $w(m G)=w(G) \geq$ $\omega$ one imposes the stronger condition $w(m G)=w(G)>\omega$, which rules out all second countable abelian groups (in the discrete case all countable abelian groups). Since this is somewhat restrictive from the point of view of the current paper, we adopt this slight modification here.

It is worth to observe also the following relation of the divisible weight with a previously defined cardinal invariant of abelian groups. Indeed, in [14] (see [8, §35]) the final rank of an abelian $p$-group $G$, for $p$ a prime, was defined as

$$
\operatorname{fin} r(G)=\inf _{n \in \mathbb{N}} r_{p}\left(p^{n} G\right)
$$

where $r_{p}$ denotes the $p$-rank. Since fin $r(G)=w_{d}(G)$ in case $r_{p}\left(p^{n} G\right)$ is infinite for every $n \in \mathbb{N}_{+}$, the divisible weight can be viewed as a natural generalization of the final rank to all (topological) abelian groups.

2010 MSC: primary 22C05, 22A05; secondary 20K45, 54H11, 54D30

Keywords: abelian group, compact abelian group, torsion theory, bounded abelian group, w-divisible group, divisible weight, factorization theorem 
In Section 2 we recall known general properties of w-divisible abelian groups and we prove some new results, paying a special attention to the discrete case.

Let $w \mathcal{D}$ be the class of all w-divisible discrete abelian groups; it is worth underlining immediately that this class contains those of all torsion-free abelian groups and of all divisible abelian groups. Since these classes are respectively the torsion-free class and the torsion class of two different torsion theories, following [2, 11] we recall that a pair $(\mathcal{T}, \mathcal{F})$ of non-empty subclasses of the category of (topological) abelian groups is called a torsion theory if and only if:

(a) $\operatorname{Hom}(T, F)=0$ for every $T \in \mathcal{T}$ and every $F \in \mathcal{F}$;

(b) $\mathcal{T}$ and $\mathcal{F}$ are maximal with respect to (a).

Moreover,

(i) $\mathcal{T}$ is a torsion class if and only if it is closed under quotients, coproducts and extensions;

(ii) $\mathcal{F}$ is a torsion-free class if and only if it is closed under subobjects, products and extensions.

Every torsion theory $\mathfrak{t}=(\mathcal{T}, \mathcal{F})$ can be obtained by means of a radical $\mathbf{r}$ as follows (see [2]). A preradical $\mathbf{r}$ associates to every abelian group $G$ its subgroup $\mathbf{r}(G)$ in such a way that $f(\mathbf{r}(G)) \subseteq \mathbf{r}(G)$ for every homomorphism of abelian groups $f: G \rightarrow H$. The preradical $\mathbf{r}$ is a radical if $\mathbf{r}(G / \mathbf{r}(G))=0$ for every abelian group $G$. It is an idempotent preradical if in addition $\mathbf{r}(\mathbf{r}(G))=\mathbf{r}(G)$ for every abelian group $G$.

If $\mathbf{r}$ is an idempotent preradical, let $\mathcal{T}_{\mathbf{r}}=\{G: \mathbf{r}(G)=G\}$ and $\mathcal{F}_{\mathbf{r}}=\{G: \mathbf{r}(G)=0\}$. It is known that if $\mathbf{r}$ is an idempotent radical, then $\mathfrak{t}_{\mathbf{r}}=\left(\mathcal{T}_{\mathbf{r}}, \mathcal{F}_{\mathbf{r}}\right)$ is a torsion theory. For every torsion theory $\mathfrak{t}=(\mathcal{T}, \mathcal{F})$ one associates an idempotent radical as follows. For every abelian group $G$ there exists a unique greatest subgroup $G_{\mathfrak{t}}$ of $G$ such that $G_{\mathfrak{t}} \in \mathcal{T}$ and $G / G_{\mathfrak{t}} \in \mathcal{F}$ (with $G_{\mathfrak{t}}=\sum\{T \subseteq G: T \in \mathcal{T}\}$ ). Moreover, the correspondence $\mathbf{r}_{\mathfrak{t}}: G \mapsto G_{\mathfrak{t}}$ is an idempotent radical (if $\mathfrak{t}$ is hereditary, then $\mathbf{r}_{\mathfrak{t}}$ is hereditary too). So starting from an idempotent radical $\mathbf{r}$, we have that $\mathbf{r}_{t_{\mathbf{r}}}=\mathbf{r}$. In other words, there is a bijective correspondence between all idempotent radicals and all torsion theories.

The torsion-free abelian groups and the torsion abelian groups form the fundamental example of torsion theory; indeed, the torsion abelian groups are the torsion class $\mathcal{T}_{\mathbf{t}}$ of this torsion theory, while the torsion-free abelian groups form the torsion-free class $\mathcal{F}_{\mathbf{t}}$; here $\mathbf{t}$ is the radical associating to every abelian group $G$ its torsion subgroup $t(G)$. Furthermore, recall that an abelian group is reduced if it has no non-trivial divisible subgroup; the reduced abelian groups and the divisible abelian groups form another torsion theory, where the divisible abelian groups are the torsion class $\mathcal{T}_{\mathbf{d}}$ and the reduced abelian groups are the torsion-free class $\mathcal{F}_{\mathbf{d}}$; here $\mathbf{d}$ is the radical associating to every abelian group $G$ its maximal divisible subgroup $d(G)$.

Going back to the class $w \mathcal{D}$ of all w-divisible discrete abelian groups, note again that $w \mathcal{D}$ contains the torsion-free class $\mathcal{F}_{\mathbf{t}}$ of the first named torsion theory and the torsion class $\mathcal{T}_{\mathbf{d}}$ of the second one. What remains outside of $w \mathcal{D}$ are the bounded torsion abelian groups; more precisely, if $\mathcal{B}$ denotes the class of all bounded torsion abelian groups, then $w \mathcal{D}$ and $\mathcal{B}$ have trivial intersection. So now we discuss the properties of the pair $(\mathcal{B}, w \mathcal{D})$ with respect to those of torsion theories.

The choice of this specific pair is corroborated by the forthcoming discussion. Indeed, in analogy to the concept of reduced abelian groups versus divisible abelian groups, one would like to consider that of w-reducibility versus w-divisibility; in this direction one can define a topological abelian group $G$ to be w-reduced if $G$ does not contain any non-trivial w-divisible subgroup. It is easy to see that the w-reduced abelian groups are precisely the bounded torsion ones (see Lemma 2.7).

In the following table we summarize the stability properties of the classed $w \mathcal{D}$ (see Section 2 ) and $\mathcal{B}$, to better understand the situation of the pair $(\mathcal{B}, w \mathcal{D})$, which is not a torsion theory, but appears to be something like a torsion theory with the stability properties mixed in some way. 


\begin{tabular}{|c|ccccc|}
\hline & extensions & subobjects & quotients & products & coproducts \\
\hline$w \mathcal{D}$ & yes & no & no & yes & yes \\
$\mathcal{B}$ & yes & yes & yes & no & no \\
\hline
\end{tabular}

In particular, this shows clearly that $w \mathcal{D}$ is not a torsion-free class neither a torsion class of some torsion theory, and analogously $\mathcal{B}$ cannot be a torsion class. On the other hand, it should be desirable to introduce some general notion of "mixed" torsion theory that captures the pair $(\mathcal{B}, w \mathcal{D})$. Indeed, with respect to the pair $(\mathcal{B}, w \mathcal{D})$ we discuss and prove a decomposition theorem. Going into the details, in [9] the following decomposition theorem was proved; we state it using the terminology of w-divisibility.

Theorem 1.2. [9, Lemma 4.1] Let $G$ be a discrete abelian group. Then $G=G_{t o r} \oplus G_{d}$, where $G_{t o r}$ is bounded torsion and $G_{d}$ is w-divisible.

In the dual situation (with respect to Pontryagin duality), this factorization was already pointed out in [5, Remark 4.8] in the case of abelian pro- $p$ groups. For a discrete abelian group $G$, we denote by $\widehat{G}$ the Pontryagin dual of $G$; in this case $\widehat{G}$ is a compact abelian group. Moreover, $G$ is bounded torsion precisely when $\widehat{G}$ is bounded torsion and $G$ is w-divisible precisely when $\widehat{G}$ is w-divisible. So in Section 3 we prove the following decomposition theorem for compact abelian groups $K$. Indeed, we see that such a group $K$ can be split as a direct product of a bounded torsion compact abelian group and a w-divisible compact abelian group. As explained above, it is worth to observe clearly that by Pontryagin duality our theorem is equivalent to [9, Lemma 4.1] (here Theorem 1.2).

Theorem 1.3. Let $K$ be a compact abelian group. Then $K$ splits topologically in a direct product $K=K_{\text {tor }} \times K_{d}$, where $K_{\text {tor }}$ is a bounded torsion compact abelian group, while the compact abelian group $K_{d}$ is $w$-divisible.

As an application of Theorem 1.3 we find the same factorization for some non-compact topological abelian groups, for example for an abelian group endowed with its Bohr topology (see Corollary 3.5). One of the reasons for which the factorization is available for the Bohr topology is that it is a functorial topology, so in particular it distributes on finite direct products (see for example [8] for more details on functorial topologies).

Let us thickly underline at this point that the factorization $K=K_{\text {tor }} \times K_{d}$ of Theorem 1.3 is not unique, and the subgroup $K_{d}$ is w-divisible, but it is not necessarily the greatest w-divisible subgroup of $K$ (see Example 4.1).

In particular, this shows that to the pair $(\mathcal{B}, w \mathcal{D})$ it is not possible to associate a radical as in the case of torsion theories described above. Indeed, for example the sum of all w-divisible subgroups of an abelian group $G$ is not necessarily w-divisible.

We leave open the following problem, asking for a possible generalization of the factorization given by Theorem 1.3 and up to where this extension is available.

Problem 1.4. (a) Find a topological abelian group $G$ admitting no factorization as in Theorem 1.3 .

(b) Can Theorem 1.3 be extended to all locally compact abelian groups?

In the final Section 4 we collect some applications of Theorem 1.3 to the problem of finding dense free abelian subgroups of compact groups, recently studied from different point of views in [1] and [3]. 


\section{$2 \quad$ w-Divisible groups}

We start this section recalling the general properties of w-divisible abelian groups proved in [5].

Lemma 2.1. [5, Lemma 3.1] Let $G=\prod_{i \in I} G_{i}$ where each $G_{i}$ is a topological group. If $G_{i}$ is w-divisible for every $i \in I$, then $G$ is w-divisible.

The quotient of a w-divisible abelian group need not be w-divisible; indeed, take for example $\mathbb{J}_{p}^{\mathfrak{c}}$ and its quotient $\mathbb{Z}(p)^{\mathfrak{c}}$, where $p$ is a prime and $\mathbb{J}_{p}$ denotes the $p$-adic integers and $\mathbb{Z}(p)$ the cyclic group with $p$ many elements.

Lemma 2.2. [5, Claim 3.2] Let $G$ be a topological abelian group.

(a) There exists $m \in \mathbb{N}_{+}$such that $w_{d}(G)=w(m G)=w_{d}(m G)$; in particular, $m G$ is $w$-divisible.

(b) If $H$ is a subgroup of $G$, then $w_{d}(G) \geq w_{d}(H)$ and $w_{d}(G) \geq w_{d}(G / H)$;

(c) If $G$ is precompact and $L$ is a precompact continuous image of $G$, then $w_{d}(G) \geq w_{d}(L)$.

Lemma 2.3. [5, Claim 3.6] If $n \in \mathbb{N}_{+}, G_{1}, \ldots, G_{n}$ are topological abelian groups and $G=G_{1} \times \ldots \times G_{n}$, then $w_{d}(G)=\max \left\{w_{d}\left(G_{1}\right), \ldots, w_{d}\left(G_{n}\right)\right\}$. In particular, $G$ is w-divisible if and only if $G_{i}$ is w-divisible for some $i=1, \ldots, n$ such that $w(G)=w\left(G_{i}\right)$.

This works with a finite number of groups but fails to be true in general taking infinitely many groups; indeed, for example take $K=\prod_{p} \mathbb{Z}(p)^{\omega_{1}}$ with $K_{p}=\mathbb{Z}(p)^{\omega_{1}}$, then $w_{d}(K)=w(K)$ while $w_{d}\left(K_{p}\right)=1$ for every prime $p$.

We state explicitly some clear properties of w-divisible discrete abelian groups that apply in the proof of Theorem 1.3 given below. Note that the first two in (a) and (b) follow directly from the definitions and from the properties of the weight. The statement in (c) is a corollary of (b) since for an abelian group $G$ the quotient $G / t(G)$ is torsion-free, hence w-divisible. Furthermore, the property in (d) can be given in a stronger form, requiring only the subgroup of maximum cardinality to be w-divisible.

Lemma 2.4. Let $G$ be a discrete abelian group.

(a) If $G=\bigoplus_{i \in I} G_{i}$ for a family $\left\{G_{i}: i \in I\right\}$ of subgroups of $G$, and $G_{i}$ is w-divisible for every $i \in I$, then $G$ is w-divisible.

(b) If $H$ is a w-divisible subgroup of $G$ and $G / H$ is w-divisible, then $G$ is w-divisible.

(c) If $t(G)$ is w-divisible, then $G$ is w-divisible.

(d) If the subgroups $H_{1}, \ldots, H_{n}$ of $G$ are w-divisible, then also $H_{1}+\ldots+H_{n}$ is w-divisible.

In spite of item (a) and unlike the property of divisibility, the weaker property of w-divisibility is not preserved under taking direct limits. Moreover, note that if $G$ is a w-divisible abelian group and $B$ is a bounded torsion abelian group such that $w(B) \leq w(G)$ (so $|B| \leq|G|$ in case the groups are discrete), then $G \times B$ is still w-divisible.

Remark 2.5. Let $G$ be a discrete abelian group. Item (c) of Lemma 2.4 states that $G$ is w-divisible if its torsion subgroup $t(G)$ is w-divisible. Write now $t(G)=\bigoplus_{p} t_{p}(G)$, where $t_{p}(G)$ is the $p$-torsion part of $G$ for every prime $p$. By Lemma 2.4 (a) we have that $t(G)$ is w-divisible if $t_{p}(G)$ is w-divisible for every prime $p$. Clearly, the converse implication does not hold true in general. On the other hand, the Pontryagin dual of a torsion abelian group $G$ is a totally disconnected compact abelian group $K$, and the w-divisibility of such $K$ is discussed in details in [5, §4] in terms of the topological $p$-components $K_{p}$ (which correspond to the $p$-torsion parts of $G$ ) since $K=\prod_{p} K_{p}$. 
The following easy relation between the divisible weight of a compact abelian group and that of its discrete Pontryagin dual group was already observed in [5].

Lemma 2.6. Let $G$ be a discrete abelian group. Then $w_{d}(G)=w_{d}(\widehat{G})$.

Consequently, $G$ is w-divisible if and only if $\widehat{G}$ is w-divisible.

Proof. Let $K=\widehat{G}$. Since $n G \cong \widehat{n K}$ for every $n \in \mathbb{N}_{+}$, one has $|n G|=w(n K)$, hence the conclusion follows.

In the following proposition we see that the bounded torsion abelian groups, that clearly are precisely the w-reduced abelian groups, coincide also with the abelian groups admitting no non-trivial w-divisible quotient.

Proposition 2.7. Let $G$ be a discrete (or compact) abelian group. Then the following conditions are equivalent:

(a) G has no non-trivial w-divisible subgroups (i.e., $G$ is w-reduced);

(b) $G$ is bounded torsion;

(c) G has no non-trivial w-divisible quotient.

Proof. We prove the result for a discrete abelian group $G$, the compact case follows by Pontryagin duality and applying Lemma 2.6 .

$(\mathrm{b}) \Rightarrow(\mathrm{a})$ and $(\mathrm{b}) \Rightarrow(\mathrm{c})$ are trivial. To see that $(\mathrm{a}) \Rightarrow(\mathrm{b})$ use the fact that there exists always $m \in \mathbb{N}_{+}$ such that $m G$ is w-divisible by Lemma 2.2 (a), so $m G=0$ by hypothesis.

(c) $\Rightarrow$ (b) Assume that $m G \neq 0$ for every $m \in \mathbb{N}_{+}$. We find a non-trivial w-divisible quotient of $G$.

By our assumption, for every $m \in \mathbb{N}_{+}$there exists $g_{m} \in G$ such that $m g_{m} \neq 0$. Let $H=\left\langle g_{m}\right.$ : $\left.m \in \mathbb{N}_{+}\right\rangle$; in particular, $H$ is countably infinite and is not bounded torsion. By Zorn's Lemma there exists a subgroup $M$ of $G$ maximal with respect to the property $M \cap H=0$. Let $q: G \rightarrow G / M$ be the canonical projection. Then $q(H) \cong H$ as $M \cap H=0$, and by the maximality of $M$ in $G$ we have that $q(H)$ is essential in $G / M$. This implies that $G / M$ is countably infinite. We see that $m(G / M)$ has the same cardinality of $G / M$ for every $m \in \mathbb{N}_{+}$. To this end let $m \in \mathbb{N}_{+}$; as $|G / M| \geq|m(G / M)|$ it suffices to see that $m(G / M)$ is infinite. This holds true as $m H$ is an infinite subgroup of $m(G / M)$, since $H$ is not bounded torsion.

Observe that the stronger property contained in the equivalence $(\mathrm{a}) \Leftrightarrow(\mathrm{c})$ does not hold for the classical reduced abelian groups. Indeed, there exist examples of reduced abelian groups with divisible quotients, such as $\mathbb{Z}^{(\omega)}$ that admits a surjective homomorphism onto $\mathbb{Q}$. On the other hand, if an abelian group $G$ has no divisible quotients, then it has no divisible subgroups. To complete this comment on Proposition 2.7, we add that abelian groups $G$ with no divisible quotients were characterized in [6] for example as those abelian groups admitting no surjective homomorphism on $\mathbb{Z}\left(p^{\infty}\right)$ for any prime $p$, where $\mathbb{Z}\left(p^{\infty}\right)$ denotes the Prüfer group.

We show now that the class of w-divisible abelian groups contains that of monothetic groups. In the compact case this can be considered as a particular case of Theorem 1.3 .

Proposition 2.8. Let $G$ be a monothetic group. Then $G$ is w-divisible.

Proof. Let $x$ be a topological generator of $G$, that is $G$ coincides with the closure of $C=\langle x\rangle \cong \mathbb{Z}$; in particular, $w(C)=w(G)$ as $C$ is dense in $G$. Let $m \in \mathbb{N}_{+}$; we have to verify that $w(m G)=w(G)$. Since $m C$ is dense in $m G$, we have $w(m C)=w(m G)$. Moreover, $m C$ has finite index in $C$, so also the closure $\overline{m C}$ of $m C$ in $C$ has finite index in $C$, so $\overline{m C}$ is open in $C$ and therefore $w(m C)=w(\overline{m C})=w(C)$. now we can conclude that $w(m G)=w(m C)=w(C)=w(G)$, as wanted. 
The last part of this section is dedicated to the w-divisibility of three specific functorial topologies. We start recalling that the Bohr topology of an abelian group $G$ is the initial topology of all homomorphisms $G \rightarrow \mathbb{T}=\mathbb{R} / \mathbb{Z}$, namely, the characters of $G$, where $\mathbb{T}$ is equipped with the compact quotient topology of $\mathbb{R} / \mathbb{Z}$; we denote by $G^{\sharp}$ the abelian group $G$ endowed with its Bohr topology, which is in particular a precompact abelian group.

We give now the following consequence of the nice property of the Bohr topology to have the maximal possible weight, that is

$$
w\left(G^{\sharp}\right)=2^{|G|}
$$

for any abelian group $G$. The second part of the proposition is an independence result in ZFC.

Proposition 2.9. Let $G$ be an abelian group. If $G$ is w-divisible then $G^{\sharp}$ is w-divisible.

Moreover, the validity of the converse implication is independent in $Z F C$.

Proof. By the equality in (2.1) and by hypothesis, for any $m \in \mathbb{N}_{+}$we have

$$
w\left(m G^{\sharp}\right)=2^{|m G|}=2^{|G|}=w\left(G^{\sharp}\right),
$$

hence $w_{d}\left(G^{\sharp}\right)=w\left(G^{\sharp}\right)$.

Assume GCH (Generalized Cardinal Hypothesis). If $G^{\sharp}$ is w-divisible, then by (2.1) we have that

$$
2^{|m G|}=w\left(m G^{\sharp}\right)=w\left(G^{\sharp}\right)=2^{|G|}
$$

for every $m \in \mathbb{N}_{+}$. By GCH we can conclude that $|m G|=|G|$ for every $m \in \mathbb{N}_{+}$, that is $G$ is w-divisible.

Assume now LH (Lusin Hypothesis), that implies the equality $2^{\omega}=2^{\omega_{1}}$. Let $G=\bigoplus_{\omega} \mathbb{Z} \oplus \bigoplus_{\omega_{1}} \mathbb{Z}(2)$. Then $G^{\sharp}$ is w-divisible, while $G$ is not w-divisible. Indeed, $|G|=\omega_{1}$ and $|2 G|=\omega$, so $G$ is not wdivisible. On the other hand, by 2.1) we have $w\left(m G^{\sharp}\right)=w\left(G^{\sharp}\right)$ for every $m \in \mathbb{N}_{+}$, hence $G^{\sharp}$ is w-divisible.

We consider now other two functorial topologies. Indeed, for an abelian group $G$, the profinite topology $\gamma_{G}$ has all finite-index subgroups as a base of the neighborhoods of 0 , while the natural topology $\nu_{G}$ has the countable family of subgroups $\left\{m G: m \in \mathbb{N}_{+}\right\}$as a base of the neighborhoods of 0 .

It is worth to recall that in [4, Theorem 2.13] it was proved that for any abelian group the profinite topology is the infimum of the natural topology and the Bohr topology. Moreover, the profinite topology coincides with the Bohr topology precisely when the abelian group is bounded torsion.

We recall the following two computations of the weight respectively of the natural and the profinite topologies given in [4, Theorem 3.6 and Theorem 3.3]. We first remind that an abelian group $G$ is residually finite if $G$ is isomorphic to a subgroup of a direct product of finite abelian groups; moreover, $G$ is residually finite precisely when $\gamma_{G}$ is Hausdorff. In this case we have

$$
w\left(G, \nu_{G}\right)=\omega \cdot \sup \{|G / p G|: p \text { prime }\}
$$

and

$$
w\left(G, \gamma_{G}\right)=\omega \cdot \sup \left\{2^{|G / p G|}: p \text { prime }\right\} .
$$

Using these equalities we can prove the counterpart of Proposition 2.9 also for the natural and the profinite topology, assuming the abelian group $G$ to be also torsion-free, so necessarily w-divisible.

Proposition 2.10. Let $G$ be an infinite residually finite torsion-free abelian group. Then $\left(G, \nu_{G}\right)$ and $\left(G, \gamma_{G}\right)$ are w-divisible. 
Proof. Let $m \in \mathbb{N}_{+}$. Since $G$ is torsion-free, we have that $|G / p G|=|m G / p m G|$ for every prime $p$. By the equality in 2.2 and by hypothesis,

$$
w\left(m G, \nu_{G}\right)=\omega \cdot \sup \{|m G / p m G|: p \operatorname{prime}\}=\omega \cdot \sup \{|G / p G|: p \operatorname{prime}\}=w\left(G, \nu_{G}\right) .
$$

Since $m \in \mathbb{N}_{+}$was chosen arbitrarily, we conclude that $w_{d}\left(G, \nu_{G}\right)=w\left(G, \nu_{G}\right)$.

By the equality in (2.3) and by hypothesis,

$$
w\left(m G, \gamma_{G}\right)=\omega \cdot \sup \left\{2^{|m G / p m G|}: p \operatorname{prime}\right\}=\omega \cdot \sup \left\{2^{|G / p G|}: p \operatorname{prime}\right\}=w\left(G, \gamma_{G}\right) .
$$

Since $m \in \mathbb{N}_{+}$was chosen arbitrarily, we conclude that $w_{d}\left(G, \gamma_{G}\right)=w\left(G, \gamma_{G}\right)$.

\section{Splitting compact abelian groups}

We define now a special kind of direct products and direct sums of cyclic $p$-groups, that give rise to direct products of cyclic $p$-groups which are $w$-divisible.

Definition 3.1. Let $p$ be a prime and let $\alpha_{n}$ be cardinals for $n \in \mathbb{N}_{+}$. A direct sum $\bigoplus_{n \in \mathbb{N}_{+}} \mathbb{Z}\left(p^{n}\right)^{\left(\alpha_{n}\right)}$ or a direct product $\prod_{n \in \mathbb{N}_{+}} \mathbb{Z}\left(p^{n}\right)^{\alpha_{n}}$ is called uniform if for every $n \in \mathbb{N}_{+}$there exist infinitely many $m \geq n$ such that $\alpha_{m} \geq \alpha_{n}$.

Consider the product $K=\prod_{n \in \mathbb{N}_{+}} \mathbb{Z}\left(p^{n}\right)^{\alpha_{n}}$ and set

$$
\alpha_{K}=\sup _{n \in \mathbb{N}_{+}} \alpha_{n}
$$

For every $n \in \mathbb{N}_{+}$, let $\beta_{n}=\sup _{m \geq n} \alpha_{m}$, and note that this is a descending sequence:

$$
\alpha_{K}=\beta_{1} \geq \beta_{2} \geq \ldots \geq \beta_{n} \geq \beta_{n+1} \geq \ldots ;
$$

in particular $\beta_{n} \leq \alpha_{K}$ for every $n \in \mathbb{N}_{+}$. So let also

$$
\gamma_{K}=\min _{n \in \mathbb{N}_{+}} \beta_{n}
$$

clearly, in general $\gamma_{K} \leq \alpha_{K}$.

In case the product $K$ is equipped with the product topology, then $\alpha_{K}=w(K)$, moreover $\beta_{n}=$ $w\left(p^{n-1} K\right)$ for every $n \in \mathbb{N}_{+}$and so $\gamma_{K}=w_{d}(K)$. So we have the following

Lemma 3.2. Let $p$ be a prime, let $\alpha_{n}$ be cardinals for $n \in \mathbb{N}_{+}$, and let $K=\prod_{n \in \mathbb{N}_{+}} \mathbb{Z}\left(p^{n}\right)^{\alpha_{n}}$. Then the following conditions are equivalent:

(a) $K$ is uniform;

(b) $\gamma_{K}=\alpha_{K}$;

(c) $K$ is w-divisible.

Before giving the proof of Theorem 1.3 , we need to prove the following particular case.

Lemma 3.3. Let $p$ be a prime, let $\alpha_{n}$ be cardinals for $n \in \mathbb{N}_{+}$, and let $K=\prod_{n \in \mathbb{N}_{+}} \mathbb{Z}\left(p^{n}\right)^{\alpha_{n}}$. Then there exists $m \in \mathbb{N}_{+}$such that

$$
K=K_{\text {tor }} \times K_{d},
$$

where $K_{\text {tor }}=\prod_{n=1}^{m} \mathbb{Z}\left(p^{n}\right)^{\alpha_{n}}$ is bounded torsion and $K_{d}=\prod_{n=m+1}^{\infty} \mathbb{Z}\left(p^{n}\right)^{\alpha_{n}}$ is w-divisible. 
Proof. In the above notations, there exists $m \in \mathbb{N}_{+}$such that $\gamma_{K}=\beta_{m}$, so that for every $n>m$ in $\mathbb{N}_{+}$one has $\beta_{n}=\beta_{m}$, i.e., there exist infinitely many $k \in \mathbb{N}_{+}$such that $\alpha_{k} \geq \alpha_{n}$. This $m$ works, as $K_{d}=\prod_{n=m+1}^{\infty} \mathbb{Z}\left(p^{n}\right)^{\alpha_{n}}$ is uniform by construction, hence w-divisible in view of Lemma 3.2 .

Now we recall another particular case of Theorem 1.3 , pointed out without proof already in [5, Remark 4.8]. For reader's convenience we show how to deduce it from [5, Remark 4.5].

Lemma 3.4. Let $p$ be a prime. Theorem 1.3 holds true for every abelian pro-p group $K$.

Proof. Let $X=\widehat{K}$ be the discrete Pontryagin dual of $K$, which is a $p$-group. According to [5, Remark 4.5], there exists a subgroup $B$ of $X$, such that

(a) $B=\bigoplus_{n \in \mathbb{N}_{+}} \mathbb{Z}\left(p^{n}\right)^{\left(\alpha_{n}\right)}$ for some cardinals $\alpha_{n}$ with $n \in \mathbb{N}_{+}$;

(b) for every $m \in \mathbb{N}_{+}$, letting

$$
B_{1, m}=\bigoplus_{n=1}^{m} \mathbb{Z}\left(p^{n}\right)^{\left(\alpha_{n}\right)} \text { and } B_{2, m}=\bigoplus_{n=m+1}^{\infty} \mathbb{Z}\left(p^{n}\right)^{\left(\alpha_{n}\right)},
$$

the group $X$ factorizes as

$$
X=X_{m} \oplus B_{1, m}
$$

where $X_{m}=p^{m} X+B_{2, m}$.

By Lemma 3.3 applied to the Pontryagin dual $\prod_{n \in \mathbb{N}_{+}} \mathbb{Z}\left(p^{n}\right)^{\alpha_{n}}$ of $B$, we can choose $m \in \mathbb{N}_{+}$in such a way to have the "tail" $B_{2, m}$ of the direct sum $B=B_{1, m} \oplus B_{2, m}$ to be w-divisible. Since $p^{s} B_{2, m}$ remains w-divisible for every $s \in \mathbb{N}$, by choosing $m$ eventually bigger, one can achieve to have simultaneously $p^{m} X$ w-divisible. Then $X_{m}=p^{m} X+B_{2, m}$ is w-divisible by Lemma 2.4(d) as a sum of two w-divisible subgroups.

Hence, the factorization in $(3.1)$ provides a factorization $K=K_{t o r} \times K_{d}$ of $K$, where $K_{t o r}=\widehat{B}_{1, m}$ is bounded torsion and $K_{d}=\widehat{X}_{m}$ is w-divisible by Lemma 2.6 .

We are now in position to prove our decomposition theorem.

Proof of Theorem 1.3. Assume first that $K$ is totally disconnected. Then $K$ splits in a direct product $K=\prod_{p} K_{p}$, where $K_{p}$ is a pro- $p$ group for every prime $p$. By Lemma 3.4. $K_{p}=B_{p} \times K_{p}^{*}$ where $B_{p}$ is bounded torsion and $K_{p}^{*}$ is w-divisible. Then $K^{*}=\prod_{p} K_{p}^{*}$ is still w-divisible by Lemmas 2.4(a) and 2.6. Let $B=\prod_{p} B_{p}$ and let $p^{k_{p}}$ be the exponent of $B_{p}$ for every prime $p$. If $B$ is w-divisible, then $K=B \times K^{*}$ is w-divisible too by Lemmas 2.4(a) and 2.6, so we are done.

Assume now that $B$ is not w-divisible, so $w_{d}(B)<w(B)$. By Lemma 2.2(a) there exists $m \in \mathbb{N}_{+}$ such that $m B$ is w-divisible. Denote by $\pi$ the finite set of prime divisors of $m$ and let

$$
B^{\prime}=\prod_{p \in \pi} B_{p} \text { and } B^{\prime \prime}=\prod_{p \in \mathbb{P} \backslash \pi} B_{p}
$$

Taking eventually a power of $m$ in place of $m$, one can achieve to have $m B^{\prime}=0$, i.e., $B^{\prime}=B[m]$, where $B[m]=\{x \in B: m x=0\}$. Moreover, $m B^{\prime \prime}=B^{\prime \prime}$. Hence, from $B=B^{\prime} \times B^{\prime \prime}$ one concludes that $m B^{\prime \prime}=B^{\prime \prime}=m B$ is w-divisible. Therefore, $K_{d}=B^{\prime \prime} \times K^{*}$ is w-divisible by Lemmas 2.4(a) and 2.6, and we get the desired factorization

$$
K=B^{\prime} \times\left(m B \times K^{*}\right)=B^{\prime} \times\left(B^{\prime \prime} \times K^{*}\right)=B[m] \times K_{d} .
$$

This concludes the totally disconnected case. 
Consider now the general case and the totally disconnected quotient $K / c(K)$. According to the above argument, $K / c(K)$ splits in a direct product $K / c(K)=B \times D$, where $B=(K / c(K))_{\text {tor }}$ is bounded torsion and $D=(K / c(K))_{d}$ is w-divisible.

Let $q: K \rightarrow K / c(K)$ be the quotient map, and let $K_{0}=q^{-1}(B)$. Then the compact group $K_{0}$ has $c\left(K_{0}\right)=c(K)$ and $K_{0} / c\left(K_{0}\right) \cong B$ bounded torsion. Therefore, $K_{0}$ splits, i.e., $K_{0} \cong c\left(K_{0}\right) \times K_{0} / c\left(K_{0}\right)$. Indeed, the annihilator $c\left(K_{0}\right)^{\perp}$ of $c\left(K_{0}\right)$ in the discrete abelian group $\widehat{K_{0}}$ is bounded torsion, as $c\left(K_{0}\right)^{\perp}$ is topological isomorphic to the dual group of $K_{0} / c\left(K_{0}\right)$, which is bounded torsion. It is known that a bounded torsion subgroup of a discrete abelian group splits, so in this case $\widehat{K_{0}} \cong \widehat{c\left(K_{0}\right)} \times c\left(K_{0}\right)^{\perp}$, therefore $K_{0} \cong c\left(K_{0}\right) \times K_{0} / c\left(K_{0}\right)$.

In particular, there exists a continuous section $s: K_{0} / c\left(K_{0}\right) \cong B \rightarrow K_{0}$, that is a (necessarily injective) continuous homomorphism. Then $B^{*}$ is in the range of $s$ and so it is bounded torsion, $q\left(B^{*}\right)=B$ and $K_{0}=c\left(K_{0}\right) \times B^{*}=c(K) \times B^{*}$. Let $K_{d}=q^{-1}(D)$; then obviously $K=B^{*}+K_{d}$. To see now that $B^{*} \cap K_{d}=0$, assume $b \in B^{*} \cap K_{d}$. Then $q(b) \in B \cap K_{d}^{*}=0$, so $b \in c(K)$. As $c(K) \cap B^{*}=0$, we conclude that $b=0$. Hence, we have a direct sum $K=B^{*} \times K_{d}$. To check that the product topology on $B^{*} \times K_{d}$ coincides with the topology of $K$ we recall that the product topology on $B^{*} \times K_{d}$ is the finest group topology that induces on both component $B^{*}$ and $K_{d}$ the respective given topologies. In particular, the product topology is finer than the compact topology of $K$. Since also the product topology is compact, both topologies coincide. This proves that $K=B^{*} \times K_{d}$ is the desired factorization.

The following is an easy application of Theorem 1.3 and Proposition 2.9 .

Corollary 3.5. Let $G$ be an abelian group. Then $G^{\sharp}=G_{t o r}^{\sharp} \times G_{d}^{\sharp}$, where $G_{\text {tor }}$ is bounded torsion and $G_{d}^{\sharp}$ is w-divisible.

\section{Applications and final remarks}

The following example shows that the factorization in Theorem 1.3 is not unique and that $K_{d}$ need not be the greatest w-divisible subgroup of $K$.

Example 4.1. Let $K=\mathbb{Z}_{2}^{A} \times \mathbb{T}$, where $A$ is any uncountable set. So one can consider $K_{\text {tor }}=\mathbb{Z}(2)^{A}$ and $K_{d}=\mathbb{T}$. Now $K_{B}=\mathbb{Z}(2)^{B} \times \mathbb{T}$ is w-divisible for every countable subset $B$ of $A$. Hence also $K=\mathbb{Z}(2)^{A \backslash B} \times K_{B}$ is another factorization of $K$ in the sense of Theorem 1.3 .

One can also show that there is no greatest closed w-divisible subgroup of $K$. Indeed, if $H$ is a non-trivial closed w-divisible subgroup of $K$, then $H$ necessarily contains $\mathbb{T}$, so $H$ has the form $H=L \times \mathbb{T}$, where $L$ is a closed metrizable subgroup of $\mathbb{Z}(2)^{A}$. As $K$ itself is not w-divisible, $L$ does not coincide with $\mathbb{Z}(2)^{A}$, so one can find a closed metrizable subgroup $L^{\prime}$ of $\mathbb{Z}(2)^{A}$ properly containing $L$. Then $H^{\prime}=L^{\prime} \times \mathbb{T}$ is a w-divisible subgroup of $K$ properly containing $H$.

On the other hand, considering the factorization $K=K_{t o r} \times K_{d}$ given by Theorem 1.3 , since $K_{\text {tor }}$ is bounded torsion, there exists $m \in \mathbb{N}_{+}$such that $m K \subseteq K_{d}$; therefore, one can assert that up to multiplication by a natural number $m$, the subgroup $K_{d}$ built in the proof of the theorem is the greatest w-divisible subgroup of $K$. More precisely, if $K=B \times D$, where $B$ is a closed bounded torsion subgroup and $D$ is a closed w-divisible subgroup of $K$, then $m K_{d}=m K \subseteq D$ and $m_{1} D=m_{1} K \subseteq K_{d}$, where $m_{1}$ is the exponent of $B$.

As an application of the factorization in Theorem 1.3 , and of the known result recalled in Fact 4.2 , we give now in Corollary 4.3 a new proof of the crucial implication in [3, Corollary 2] (the converse implication is immediate); the latter result is a characterization of compact abelian groups admitting a dense free abelian subgroup, that is the main topic of [3]. For a topological abelian group $G$ we 
denote by $d(G)$ the density character of $G$, that is the minimal cardinality of a dense subset of $G$; moreover, $r(G)$ denotes the free rank of an abelian group $G$.

Fact 4.2. [5, Corollary 3.9] Let $K$ be a compact abelian group. Then $r(K)=2^{w_{d}(K)}$.

This in the above fact is fundamental relation between the divisible weight and the free rank of a compact abelian group, namely a topological invariant and a purely algebraic one.

Corollary 4.3. Let $K$ be a compact abelian group. Then $K$ admits a dense free abelian subgroup if and only if $d(K) \leq r(K)$.

Proof. Let $F$ be a dense free abelian subgroup of $K$; then $d(K) \leq|F| \leq r(K)$; in particular, $d(K) \leq$ $r(K)$.

Assume now that $d(K) \leq r(K)$. By Theorem 1.3 we can factorize $K$ as $K=K_{\text {tor }} \times K_{d}$, where $K_{\text {tor }}$ is a bounded torsion compact abelian group and $K_{d}$ is a w-divisibile compact abelian group. Let $m \in \mathbb{N}_{+}$be the exponent of $K_{t o r}$. Then $w_{d}(K)=w(m K)=w\left(K_{d}\right)$ and $r(K)=r\left(K_{d}\right)$.

By Fact 4.2 we have $r\left(K_{d}\right)=2^{w_{d}\left(K_{d}\right)}=2^{w\left(K_{d}\right)}$, in particular $r\left(K_{d}\right)>w\left(K_{d}\right)$. Now a direct inductive proof (see [1]) provides a dense free abelian subgroup $F$ of $K_{d}$ such that $F=F^{\prime} \oplus F^{\prime \prime}$, with $F^{\prime}$ and $F^{\prime \prime}$ proper dense free abelian subgroups of $K_{d}$ of maximal free rank, that is $r(F)=r\left(F^{\prime}\right)=$ $r\left(F^{\prime \prime}\right)=r\left(K_{d}\right)=r(K)$.

By hypothesis $r(K) \geq d(K)$ and $d(K) \geq d\left(K_{t o r}\right)$ as $K_{t o r}$ is a quotient of $K$. So there exists a dense subgroup $D$ of $G$ such that $|D| \leq r(K)$. Consequently there exists also a surjective endomorphism $f: F \rightarrow D$ such that $f\left(F^{\prime}\right)=D$ and $f\left(F^{\prime \prime}\right)=0$. In particular, $\operatorname{ker} f \supseteq F^{\prime \prime}$ and so ker $f$ is dense in $F$, therefore the graph $\Gamma_{f}$ of $f$ is dense in $D \times F$, which is dense in $K=K_{\text {tor }} \times K_{d}$. Hence $\Gamma_{f}$ is dense in $K$. Moreover, $\Gamma_{f} \cong F$ is a free abelian subgroup of $K$ of maximal free rank.

Note that the dense free abelian subgroup $F$ of $K$ in the above corollary can be chosen of maximal rank $r(K)$, as $r(K) \geq \mathfrak{c}$ for a compact abelian group $K$.

In the next corollary we collect several applications of the factorization in Theorem 1.3 obtained in the forthcoming paper [1].

Corollary 4.4. [1] Let $K$ be a compact abelian group.

(a) If $K$ admits a dense free abelian subgroup, then there exists an independent family $\left\{F_{i}: i \in I\right\}$ of size $|K|$ of dense free abelian subgroups of $K$.

(b) There exists a pair of dense subgroups $D_{1}, D_{2}$ of $K$ with finite intersection $D_{1} \cap D_{2}$.

(c) The pair $D_{1}, D_{2}$ in item (b) can be chosen so that every dense subgroup of $K$ contains $D_{1} \cap D_{2}$.

\section{References}

[1] W. W. Comfort and D. Dikranjan, The density nucleus of a topological group, preprint.

[2] S. E. Dickson, A torsion theory for Abelian categories, Trans. Amer. Math. Soc. 121 (1966) 223-235.

[3] D. Dikranjan and A. Giordano Bruno, Compact groups with a dense free abelian subgroup, submitted.

[4] D. Dikranjan, A. Giordano Bruno, Functorial topologies and finite-index subgroups of abelian groups, Topol. Appl. 158 (17) (2011) 2391-2407.

[5] D. Dikranjan and A. Giordano Bruno, w-Divisible groups, Topology Appl. 155 (4) (2008) 252-272.

[6] D. Dikranjan and I. Prodanov, A class of compact abelian groups, Annuaire Univ. Sofia, Fac. Math. Méc. $70(1975 / 76)$ 191-206. 
[7] D. Dikranjan, Iv. Prodanov and L. Stoyanov, Topological Groups: Characters, Dualities and Minimal Group Topologies, Pure and Applied Mathematics 130, Marcel Dekker Inc., New York-Basel, 1989.

[8] L. Fuchs, Infinite abelian groups, vol. I, Academic Press New York and London, 1973.

[9] J. Galindo and S. Macario, Pseudocompact group topologies with no infinite compact subsets, J. Pure and Appl. Algebra 215 (2011) 655-663.

[10] A. Giordano Bruno, Extremal a-pseudocompact abelian groups, Forum Math. 21 (2009) 639-659.

[11] J. S. Golan, Torsion theories, Pitman Monographs and Surveys in Pure and Applied Mathematics 29; Longman Scientific \& Technical, Harlow; John Wiley \& Sons, Inc., New York 1986.

[12] E. Hewitt and K. A. Ross, Abstract Harmonic Analysis I, Grundlehren der mathematischen Wissenschaften 115, Springer, Berlin, 1963.

[13] K. H. Hofmann and S. A. Morris, The Structure of Compact Groups, de Gruyter studies in mathematics vol. 25, de Gruyter, Berlin-New York, 1998.

[14] T. Szele, On the basic subgroups of abelian p-groups, Acta Math. Acad. Sci. Hungar 5 (1954) 129-141. 